\title{
Lessons Learned from Faecal Microbiota Transplantation in Cirrhosis
}

\author{
Grace B. Hatton ${ }^{1}$. Shaolu Ran ${ }^{1}$ - Thomas H. Tranah ${ }^{1}$ • Debbie L. Shawcross ${ }^{1}$ (D) \\ Published online: 24 June 2020 \\ (C) The Author(s) 2020
}

\begin{abstract}
Purpose of Review We examine recent developments in the treatment of cirrhosis by gut microbiome manipulation specifically focusing on the phase 1 safety and feasibility trials of faecal microbiota transplantation (FMT). We interrogate the published data so far on its feasibility, safety and efficacy.

Recent Findings A large number of trials have demonstrated the efficacy of FMT in treating recurrent Clostridium difficile infection which is now considered standard of care. In cirrhosis, FMT is still being evaluated and there are a number of clinical trials underway. There are two phase 1 pilot safety studies that have been published with promising findings. However, the importance of rigorously testing donor stool for the presence of multi-drug resistant species has been highlighted and lessons have been learned.

Summary For those patients with cirrhosis, replacing an unhealthy gut microbiome with a healthy one offers a promising antibiotic-free treatment that may reduce bacterial translocation and endotoxemia.
\end{abstract}

Keywords Cirrhosis · Dysbiosis · Faecal microbiota transplantation · Gut dysbiosis · Hepatic encephalopathy

\section{Introduction}

\section{Global Chronic Liver Disease Crisis}

Cirrhosis reflects the clinical endpoint of advanced-stage liver disease, uncompromisingly characterised by a myriad of debilitating and largely irreversible symptoms. Typically, these symptoms range from immune dysregulation and infection to bleeding, fluid overload, hepatic encephalopathy (HE), endorgan failure and, ultimately, death. Treatment options are all the more limited with disease progression, and without access to liver transplantation, prognosis is invariably bleak. Equally, cirrhosis is gaining rapid traction as one of the biggest contributors to mortality worldwide [1].

Grace B. Hatton and Shaolu Ran contributed equally to this work.

This article is part of the Topical Collection on Management of the Cirrhotic Patient

Debbie L. Shawcross

debbie.shawcross@kcl.ac.uk

1 Institute of Liver Studies, Department of Inflammation Biology, School of Immunology and Microbial Sciences, Faculty of Life Sciences and Medicine, King's College London, London, UK

\section{Poor Outcomes in Chronic Liver Disease}

In the UK, $70 \%$ of patients with cirrhosis die in hospital and while one in five of those who die have had five or more admissions to hospital in the last year of life, one in five are admitted only once and die during that first admission [2]. Cirrhosis is associated with an increased incidence of infection resulting in hospitalisation and complicating hospital admissions in up to $40 \%$ of cases [3]. Infection can lead to worsening liver function and precipitate complications including variceal bleeding, HE, acute kidney injury and multiorgan failure, contributing to high mortality [4]. Patients with cirrhosis admitted to intensive care have an overall survival until hospital discharge of only $51 \%$ [5].

\section{Susceptibility to Infection and Anti-microbial Resistance in Chronic Liver Disease}

The underlying mechanisms that contribute to the development and progression of chronic liver disease are complex and encompass the development of hepatocellular inflammation, fibrosis and cirrhosis with a variable time course. This usually spans one to three decades. The gut microbiome has prime importance in the pathogenesis of cirrhosis with the 
evolution from a healthy gut microbiome to one characterised by dysregulation of gut microbial activity or 'dysbiosis' associated with the progression to end-stage cirrhosis. Dysbiosis is greater in patients with cirrhosis who develop complications related to their cirrhosis correlating with plasma endotoxin and 30-day mortality [6]. Many pre-cirrhotic liver diseases particularly alcohol and fatty liver disease [7] are associated with increased intestinal permeability and in cirrhosis, there is an imbalance between healthy and pathogenic gut bacteria with skewed microbiota populations in favour of increased numbers of pro-inflammatory and ammoniagenic species including Enterobacteriaceae, Firmicutes, Archaea and Prevotella. Bacterial translocation is a significant driver of cirrhosis-associated immune dysfunction (CAID) [8], although the mechanisms by which dysbiosis drives immune cell dysfunction remain unknown.

Patients with cirrhosis have enteric bacterial dysbiosis and translocation of bacteria across the gut epithelial barrier. This culminates in systemic inflammation and endotoxemia, inducing innate immune dysfunction, predisposing to bacterial infection. $35 \%$ of patients with cirrhosis acquire nosocomial infections compared with $5 \%$ of inpatients without cirrhosis. Patients with cirrhosis are at particularly high risk for developing antimicrobial resistance because they are frequently prescribed antibiotics (25\% are on prophylactic antibiotics), undergo multiple invasive procedures (e.g. endoscopy, recurrent paracentesis) and require recurrent hospitalisations [9, 10]. Decompensated cirrhotics have a $37 \%$ 30-day readmission rate [11]. The Canonic study [12] investigated the prevalence of multi-drug resistant organisms (MDRO) in patients with decompensated cirrhosis and acute-on-chronic liver failure in different liver centres around Europe; between one quarter and two-thirds of patients enrolled had MDRO infections, especially ESBL-Escherichia coli.

With poor outcomes following infection, the propagation of antimicrobial resistance and increasing waiting list mortality for liver transplantation, there is an urgent unmet need for approaches that focus on reducing the rate of infection and preventing recurrent hospitalisations.

\section{The Gut in Health and Chronic Liver Disease}

The gut microbiome per se is the bacterial hothouse of our digestive system, and its characteristics vary widely from person to person [13]. These variations in microbiome composition have also been repeatedly shown to link with various pathologies. Reduced species diversity and bacterial numbers are associated with inflammatory bowel disease, coeliac disease, diabetes, metabolic syndrome and even Parkinson's disease and dementia $[14,15]$. This often reduced diversity of the gut microbiome in diseased states is predominantly characterised by an overabundance of opportunistic bacterial species including Enterobacteriaceae as well as Escherichia coli, and loss of anti-inflammatory species and their metabolic products including Firmicutes and Faecalibacterium [16]. Numerous studies have also demonstrated that diet, stress, drugs and hormones play collectively strong roles in contributing to our gut microbial makeup-corroborating the microbiome's susceptibility to manipulation by the environment as much as being pre-programmed by genetics. Indeed, the functional integrity of the microbiome as we increasingly understand it is phenomenally complex; so much so that it has prompted many clinicians to advocate its status as a metabolically active tissue in itself.

Current understanding of the composition and function of the gut microbiome and how this relates to the progression and outcomes in patients with cirrhosis remains in its infancy and is based on descriptive snapshots afflicted with confounders and lacking in robust clinical validation.

The term gut 'dysbiosis' has been coined to encapsulate the perturbations in the structure of the complex commensal communities of the gut microbiome. With the onset of cirrhosis, small bowel bacterial overgrowth and a more permeable gut epithelial membrane expose the liver to immune-activating bacterial degradation products via the portal vein. This is further exacerbated by underlying portal hypertension and endothelial dysfunction, whilst portosystemic shunting increases the delivery of these bacterial degradation products to the systemic circulation, evading the reticuloendothelial system. Endotoxins activate hepatic macrophages inducing the production of proinflammatory cytokines. This can ultimately culminate in hepatic injury and systemic inflammation contributing further to immune disarray, which predisposes individuals to infection and heralds the development of decompensating complications. The evolution of gut dysbiosis has been causally linked to the pathogenesis of cirrhosis and the progression to end-stage liver disease [6]. Patients with cirrhosis also have salivary dysbiosis associated with impaired salivary defences and systemic inflammation. Salivary dysbiosis has been shown to be greater in patients with cirrhosis who developed complications necessitating hospitalisation within 90 days [17].

\section{Evidence Base for the Manipulation of the Gut Microbiome in Cirrhosis to Improve Outcomes}

Therapeutic approaches to correcting dysbiosis have, as with the causes of dysbiosis itself, been multifactorial; targeting the replenishment of lost favourable bacterial species as well as supplementing the gut with prebiotic foodstuffs to encourage butyrate-producing anti-inflammatory bacteria to thrive. There are however limits to these endeavours; treating the complications of cirrhosis with broad-spectrum antibiotics also contributes to ongoing gut dysbiosis, augmenting disruption to the normally symbiotic population of intestinal bacteria and potentially predisposing to further opportunistic infections and small bowel bacterial overgrowth $[6,18,19]$. This 
in turn adversely impacts on microbial diversity, composition and activity.

\section{Manipulation of the Gut Microbiome (Changes in Diet)}

It has been demonstrated that within a day of reaching the distal gut, a change in diet can measurably alter the gut flora and its metabolic end-products and therefore, regulation of diet itself creates an opportunity to alter the gut milieu [20]. Overall, protein intake positively correlates with microbiota diversity. In particular, consumption of plant protein has been linked to increased levels of Bifidobacteriaceae and Lactobacillaceae resulting in improved gut mucosal barrier integrity whilst animal protein has been linked to increases in abundance and activity of microorganisms capable of triggering inflammatory bowel disease, of which includes Bacteroides, Alistipes and Bilophila [20-22].

Animal models have shown that high-fat diet induces a shift in balance of gut microbiota leading to reduced levels of Lactobacillaceae and disproportionately more proinflammatory species, possibly through altered bile acid profiles $[23,24]$. The Mediterranean diet, characterised by a low intake of saturated fat and cholesterol, and a high intake of mono- and polyunsaturated fatty acid, along with complex carbohydrates and fibre, has been shown to improve cardiovascular risk and reduce inflammation [25]. This effect may in part be mediated by the increase in butyrate-producing species and decreases in pro-inflammatory species that is associated with this diet [26].

The effects of dietary habits on clinical outcomes in cirrhosis was interrogated by Bajaj et al. when they compared cirrhotic and control groups in the USA with groups from Turkey. The Turkish diet was rich in fermented milk products, coffee, tea and chocolate, all associated with increased microbiota diversity. Furthermore, it was shown that coffee, tea, vegetables and cereals were protective against 90-day rehospitalisation rates [27••]. The effects of dietary intervention on the gut flora are worth considering when managing patients with cirrhosis.

\section{Manipulation of the Gut Microbiome (Probiotics, Rifaximin)}

Beneficial modulation of the gut microbiome to improve liverrelated complications in cirrhosis has been explored through the use of indirect, non-specific means such as probiotic, prebiotic supplementation and the non-absorbable antibiotic rifaximin.

Probiotics are largely marketed as food supplements rather than licenced medicinal products but are used with the intent of conferring positive health-related effects by augmenting existing beneficial bacterial populations within the gut, thereby conferring increased microbial diversity. Equally, they may have a secondary role in inhibiting bacterial growth, including that of Pseudomonas spp. [28], methicillin-resistant
Staphylococcus aureus, Shigella spp. and Escherichia coli [29], thus potentially protecting against spontaneous bacterial peritonitis in decompensated patients. The evidence for probiotic use in treating gastrointestinal diseases remains largely conflicting; however, whilst numerous clinical trials argue that probiotic supplementation has yet to demonstrate significant benefit in such patients and namely those suffering from Traveller's diarrhoea and necrotising enterocolitis [30-32], others cite that specific strains may show promise in improving insulin sensitivity and reducing symptoms common in cirrhosis, such as hepatic encephalopathy [33, 34]. Arguably, however, one factor influencing this partial efficacy may be the intra- and inter-individual variability in gut motility, which in turn influences bacterial transit and the potential window of opportunity for beneficial bacteria species to exert their anti-inflammatory effects.

Prebiotics by comparison act as non-specific 'feedstock' for microbiota utilisation. There is some evidence to suggest their efficacy in treating dysbiosis by promoting Bifidobacterium spp. growth and which may lead to increased endogenous production of acetate and lactate, which act as substrates for beneficial butyrate-producing bacteria thought to be responsible for maintaining gut barrier integrity, such as Roseburia and Eubacterium. These species have otherwise been shown to be reduced in inflammatory bowel disease, highlighting a potential avenue for indirect modification of the gut microbiota in such diseased states by creating a preferentially favourable environment in which beneficial bacteria may thrive.

Rifaximin is a non-absorbable antibiotic which, in stark contrast to pre- and probiotic supplements, serves to nonpreferentially eliminate enteric bacterial species, destroying potentially beneficial as well as harmful strains $[35,36]$. Patients on the UK liver transplant waiting list treated with rifaximin have reduced all-cause admissions, episodes of spontaneous bacterial peritonitis and variceal bleeding, as well as reduced length of stay [37••]. Furthermore, the use of rifaximin by patients on the waiting list has been linked to reduced early allograft dysfunction following liver transplantation [38]. However, considerable concern remains regarding whether long-term antibiotic administration may drive antibiotic resistance. Indeed, in a recent study, 50\% of patients prescribed rifaximin for HE developed rifampin-resistant staphylococcal isolates after as little as 1-7 weeks following rifaximin treatment [39*•]. The question therefore is raised as to whether directly, as opposed to indirectly, modulating the gut microbiota utilising faeces from healthy donors may be a safer and more durable therapy.

\section{FMT: Origins and Uses So Far}

Direct targeting and manipulation of the gut microbiome is an increasingly promising feat of bioengineering and 
personalised medicine. One emerging strategy to address dysbiosis therapeutically is with the administration of FMT, i.e. the transplant of a homogenised filtered faecal sample from a healthy donor directly into the gastrointestinal tract of a patient with dysbiosis. The first case of FMT's use in vivo was described in 1958 [40] as an adjunct in the treatment of pseudomembranous colitis, followed by the first successful report of treatment of inflammatory bowel disease in 1989. Following this, FMT has had reported success in correcting dysbiosis, improving gut microbial diversity and reducing disease morbidity and mortality in cases of irritable bowel syndrome [41], IBD [42], metabolic syndrome [43], uro-genital infections and recurrent Clostridium difficile infection, and is now recommended in many guidelines as a therapeutic modality for the latter [44]. There is irrefutable evidence for FMT in treatment of recurrent Clostridium difficile infection; however, its benefit in first presentation Clostridium difficile infection and severe Clostridium difficile infection requires more evaluation.

In addition, there appears to be the emergence of donors that produce outstanding results in transplanted inflammatory bowel disease patients - the 'super donor' phenomenon. If we can characterise the specific factors involved, then that will allow for more targeted bacteriotherapy [45 $]$.

\section{FMT in Cirrhosis}

\section{Fresh/Frozen FMT in Cirrhosis}

End-stage liver cirrhosis inevitably leads to portal hypertension and $\mathrm{HE}$, which frequently requires hospitalisation. The aim of FMT in cirrhosis is to disrupt the pathological pathways aforementioned by restoring the gut microbiome. Some of the postulated benefits that FMT may offer in patients with cirrhosis are shown in Fig. 1.
In a pioneering open-label randomised trial of 10 patients treated with FMT via enema, Bajaj et al. showed that FMT was safe and potentially efficacious in treating HE [46••]. The group demonstrated at least part restoration of gastrointestinal bacterial diversity through treatment with FMT in cirrhotic patients following antibiotic therapy. Just one dose of rectally administered FMT was shown to reverse this dysbiosis and supports the promising use of FMT in alleviating gut microbiota disruption secondary to antimicrobials. A rational donor was selected who had a high relative abundance of Lachnospiraceae and Ruminococcacceae within a universal stool donor bank. FMT was associated with improved cognitive function. Recurrence of HE was observed in 5 of 10 patients given the standard of care but none of the 10 patients who received FMT. FMT was associated with a relative increase in the butyrate-producing species Lactobacillaceae and Bifidobacteriaceae. This study however was criticised as patients were treated with broad-spectrum antibiotics prior to FMT and the favourable impact may have been related to the antibiotic administration (not given to the standard of care arm). This would still support FMT as having utility in restoring antibiotic-induced disruption in microbial diversity and function $[47 \cdot \bullet]$.

Long-term safety and efficacy of FMT was studied within this population as the group was followed up for between 12 and 15 months (mean \pm standard deviation, $12.9 \pm 2.9$ months for FMT and $12.8 \pm 3.7$ months for standard of care). Within the standard of care group, 2 patients were excluded (1 died and 1 had a liver transplant) and within FMT group, there was 1 death. The FMT cohort had no adverse effects on long-term follow-up and, even more strikingly, there were significantly less hospitalisations compared to the standard of care group (1 in FMT group vs 10 in the standard of care group), reduced HE events ( 0 in FMT group vs 8 in standard of care group) as well as improved cognition on assessment [48••]. On microbiota analysis, although there were differences in profiles between FMT and standard of care groups, the increase in

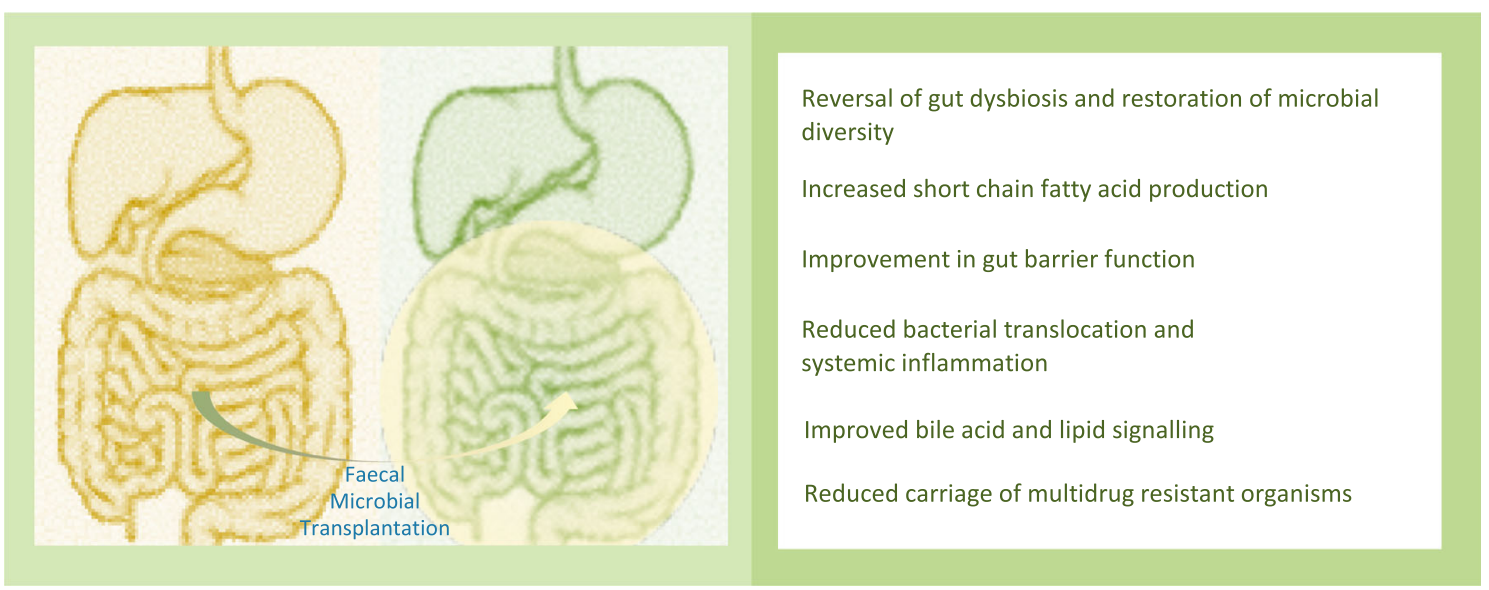

Fig. 1 Proposed benefits of faecal microbiota transplantation in cirrhosis 
Lactobacillaceae and Bifidobacteriaceae were not sustained suggesting that the recipient microbiome may not need to absolutely reflect that of the donors in order to have a beneficial effect. Further evaluation of the mechanism by which FMT produces its beneficial effects will shed insight into this matter.

$\mathrm{HE}$ is associated with a perturbed gut-liver-brain axis with the presence of brain and systemic inflammation. The potential impact of FMT on the development of neuroinflammation in cirrhotic mice as well as germ-free mice colonised by both microbiota from pre- and post-FMT cirrhotic humans as well as non-cirrhotic humans was evaluated by Liu et al. in an elegant study. Here, the investigators compared the degree of neuroinflammation in cirrhotic mice in germ-free and conventional settings that were colonised by stool collected from human cirrhotics and human healthy controls. They observed that the former showed significantly higher levels of neuroinflammation and that stool transplanted into germ-free mice from human cirrhotics who had undergone FMT attenuated the neuroinflammation [49••]. Post-FMT stool was found to have a higher abundance of Lactobacillaceae and Verrucomicrobiaceae species, the latter which has been demonstrated to protect against immune-mediated liver injury strengthening the enteric barrier.

\section{Encapsulated FMT in Cirrhosis}

Encapsulated FMT offers a more practically feasible modality of treatment and is perhaps more acceptable for patients too. Furthermore, as maintained concordance of gut flora with that of the donors has been shown to be short-lived, regular FMT therapy has been suggested as prophylaxis against HE [50].

Bajaj et al. have recently published a phase 1 study, demonstrating that oral FMT capsules are safe and well tolerated in 10 patients with cirrhosis and recurrent HE [51••]. FMT was associated with improved duodenal mucosal diversity, anti-microbial peptide expression, lipopolysaccharidebinding protein and improved cognitive performance. Microbiota analysis showed increased Ruminococceae and Verrucomicrobiaceae species. Preliminary data is encouraging and warrants further validation in larger randomised placebo-controlled trials focusing on clinical endpoints.

\section{Regulation and Problems Encountered So Far/Pitfalls}

The exact role of FMT in restoring cirrhotic gut microbial functionality in a clinical setting still remains unclear. Indeed, what is the precise composition of FMT; how is it regulated with donor selection; and what are the long-term demonstrable effects of treatment for these patients?
When FMT use is appropriate to be employed in the natural history of chronic liver disease has yet to be decided. The efficacy as well as the cost of manufacture must be considered when compared to conventional treatment modalities. A ubiquitous issue will be the innate inability to regulate the composition of FMT with the variability between donors. Furthermore, how will FMT compare to targeted bacteriophage therapy [52••]? Treatment options for patients outwith of liver transplantation are limited and therefore FMT offers an innovative alternative particularly for patients who are ineligible for liver transplantation. However, an independent issue arises in that up to $25 \%$ of cirrhotic patients are on prophylactic antibiotics. This will inherently disturb enteric flora, particularly decreasing the proportion of important short-chain fatty acid-producing species and will confound the effects of FMT.

A number of studies have illustrated a potential donordependent effect on microbial diversity and systemic metabolic response with FMT [43, 53, 54], and underpinning the difficulties in achieving treatment standardisation with donor and sample confounding. The chronology of FMT's clinical impact is also widely variable, ranging up to several years following treatment completion $[50,55,56]$.

Similarly, detailed interrogation of donor characteristics is somewhat lacking in the literature. Anand et al. studied the effects of ageing on FMT donor samples and demonstrated compositional alterations as part of their FMT microbiota analysis from donors aged over 60 years; the exact significance of this remains unclear, though did not appear to affect the clinical efficacy [57].

On a more severe note, emerging problems with FMT pertain to the inherent problems associated with regulation of donor selection, sampling and cohesion which have the potential to cause real patient harm. One such example is of emerging infections being transmitted through FMT, such as ESBL-producing E. coli [58]. Two cases have been reported of ESBL bacteraemia caused by FMT shown through genomic sequencing. One case tragically resulted in death. Subsequent investigation showed increased incidence of ESBL in stool culture of recipients from this particular donor, particularly in recipients who were treated for recurrent Clostridium difficile infection, a condition where the enteric barrier is similarly disrupted. As previously discussed, cirrhotic patients are at high risk of bacterial translocation given their state of shunting and impaired reticuloendothelial system, making them susceptible to these pathogens. FDA screening only mandated the screening for ESBL within an update on their protocol in 2019. As it is within the nature of microorganisms and viruses to mutate and alter their pathogenicity, our current screening protocols may not be able to cover all that is necessary. This carries huge clinical implications, given that these pathogens are being directly delivered into already immunosuppressed patients, leading to even higher 


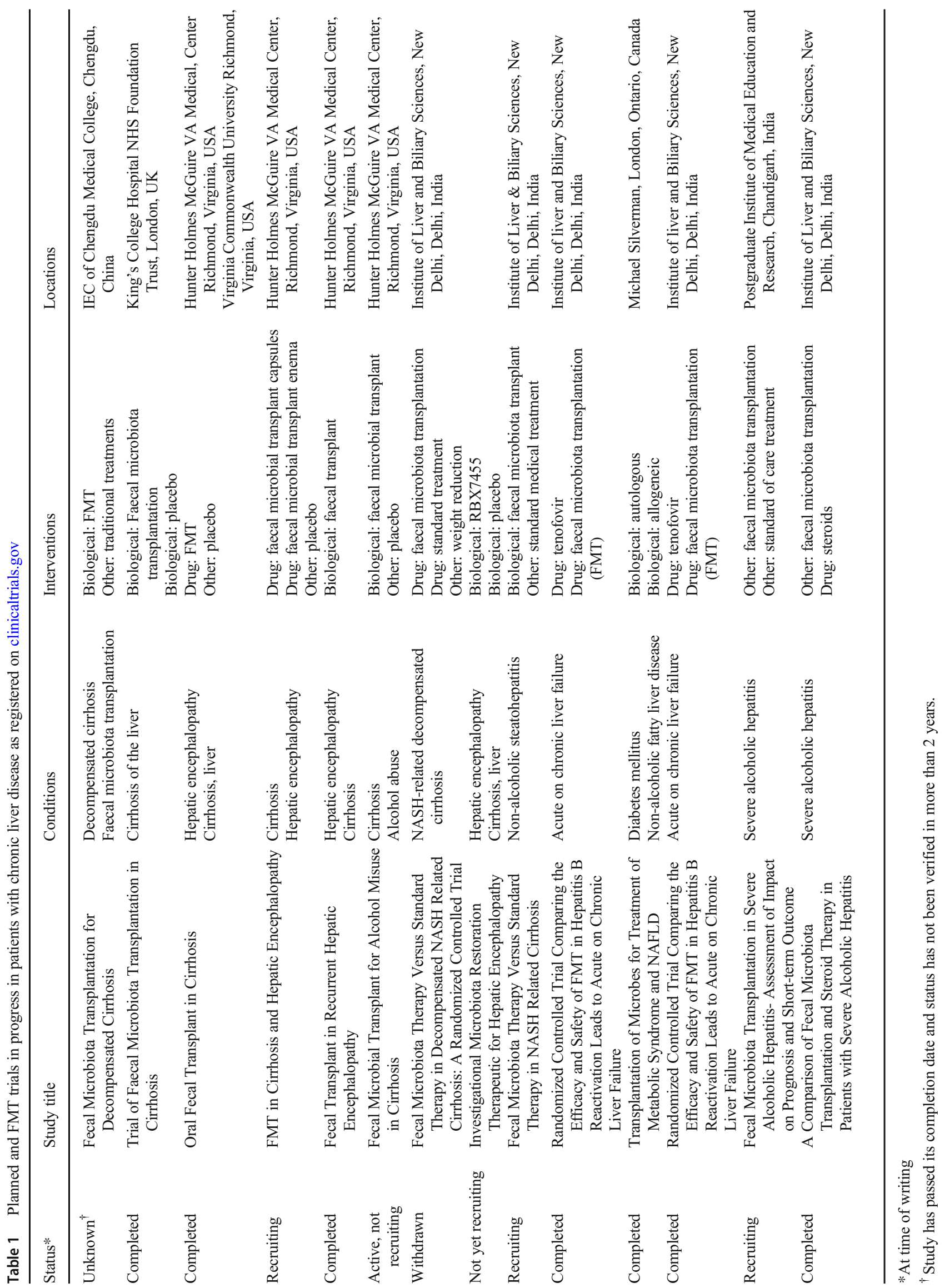


prospective rates of morbidity and mortality [59]. Furthermore, the virome is gaining increasing traction [60] and little is understood what implications FMT may pose through transmission of viruses and phages.

The UK-centric PROFIT trial [61] is one such example of a feasibility and safety study that has specifically addressed the evaluation of the mechanism of action of FMT in cirrhosis administered directly into the jejunum rather than the large bowel, with formal results due to be published in mid-2020.

FMT trials thus far have investigated by and large the preliminary safety and feasibility of FMT in diseased patients. As such, the optimal method of both manufacture and delivery of FMT remains unclear.

\section{Conclusions}

It is clear that FMT has a viable role not limited to that of gastrointestinal disease, or indeed, cirrhosis. Our emerging understanding of the gastrointestinal microbiome and its farreaching implications on health and disease has allowed for FMT to take a central role as an emerging therapy and viable alternative to conventional treatments, as well as for those patients deemed unsuitable for receipt of liver transplantation. The number of clinical trials in setup or which are currently ongoing for the treatment of chronic liver disease (Table 1) illustrates the potential interest hepatologists have in this therapeutic arena but extreme caution is advocated in trial protocolisation for this immunocompromised population.

Caveats to FMT's widespread use include its lack of regulation and donor safety as well as efficacy. Despite this, the future remains promising for the use of microbial transplantation in mainstream medicine; the role of genomic profiling and personalised medicine specific to individuals is likely to inform this directly, as well as improved donor screening and even artificial FMT creation to address these ongoing health problems.

\section{Compliance with Ethical Standards}

Conflict of Interest Professor Shawcross has participated in advisory boards for Norgine Pharmaceuticals Ltd., Shionogi and Kaleido Biosciences and has delivered paid lectures for Norgine Pharmaceuticals Ltd. and for Falk Pharma. Dr. Hatton has delivered a paid lecture for Symprove Bioscience Ltd.

Human and Animal Rights and Informed Consent This article does not contain any studies with human or animal subjects performed by any of the authors.

Abbreviations CAID, Cirrhosis-associated immune dysfunction; ESBL, Extended spectrum beta-lactamase; FMT, Faecal microbiota transplantation; HE, Hepatic encephalopathy; MDRO, Multi-drug resistant organisms
Open Access This article is licensed under a Creative Commons Attribution 4.0 International License, which permits use, sharing, adaptation, distribution and reproduction in any medium or format, as long as you give appropriate credit to the original author(s) and the source, provide a link to the Creative Commons licence, and indicate if changes were made. The images or other third party material in this article are included in the article's Creative Commons licence, unless indicated otherwise in a credit line to the material. If material is not included in the article's Creative Commons licence and your intended use is not permitted by statutory regulation or exceeds the permitted use, you will need to obtain permission directly from the copyright holder. To view a copy of this licence, visit http://creativecommons.org/licenses/by/4.0/.

\section{References}

Papers of particular interest, published recently, have been highlighted as:

- Of importance

•- Of major importance

1. Lozano R, et al. Global and regional mortality from 235 causes of death for 20 age groups in 1990 and 2010: a systematic analysis for the Global Burden of Disease Study 2010 (vol 380, pg 2095, 2012). Lancet. 2013;381(9867):628.

2. Williams R, Aspinall R, Bellis M, Camps-Walsh G, Cramp M, Dhawan A, et al. Addressing liver disease in the UK: a blueprint for attaining excellence in health care and reducing premature mortality from lifestyle issues of excess consumption of alcohol, obesity, and viral hepatitis. Lancet. 2014;384(9958):1953-97.

3. Borzio M, Salerno F, Piantoni L, Cazzaniga M, Angeli P, Bissoli F, et al. Bacterial infection in patients with advanced cirrhosis: a multicentre prospective study. Dig Liver Dis. 2001;33(1):41-8.

4. Jalan R, Fernandez J, Wiest R, Schnabl B, Moreau R, Angeli P, et al. Bacterial infections in cirrhosis: a position statement based on the EASL Special Conference 2013. J Hepatol. 2014;60(6):131024.

5. Shawcross DL, Austin MJ, Abeles RD, McPhail MJW, Yeoman $\mathrm{AD}$, Taylor NJ, et al. The impact of organ dysfunction in cirrhosis: survival at a cost? J Hepatol. 2012;56(5):1054-62.

6. Bajaj JS, Heuman DM, Hylemon PB, Sanyal AJ, White MB, Monteith $\mathrm{P}$, et al. Altered profile of human gut microbiome is associated with cirrhosis and its complications. J Hepatol. 2014;60(5):940-7.

7. Bishehsari F, Magno E, Swanson G, Desai V, Voigt RM, Forsyth $\mathrm{CB}$, et al. Alcohol and gut-derived inflammation. Alcohol Research-Current Reviews. 2017;38(2):163-71.

8. Albillos A, Lario M, Alvarez-Mon M. Cirrhosis-associated immune dysfunction: distinctive features and clinical relevance. J Hepatol. 2014;61(6):1385-96.

9. O'Brien AJ, et al. Immunosuppression in acutely decompensated cirrhosis is mediated by prostaglandin E-2. Nat Med. 2014;20(5): 522-7.

10. Fernandez J, et al. Multidrug-resistant bacterial infections in patients with decompensated cirrhosis and with acute-on-chronic liver failure in Europe. J Hepatol. 2019;70(3):398-411.

11. Volk ML, Tocco RS, Bazick J, Rakoski MO, Lok AS. Hospital readmissions among patients with decompensated cirrhosis. Am J Gastroenterol. 2012;107(2):247-52.

12. Fernandez J, et al. Prevalence and risk factors of infections by multiresistant bacteria in cirrhosis: a prospective study. Hepatology. 2012;55(5):1551-61. 
13. Huttenhower C, et al. Structure, function and diversity of the healthy human microbiome. Nature. 2012;486(7402):207-14.

14. De Cruz P, et al. Characterization of the gastrointestinal microbiota in health and inflammatory bowel disease. Inflamm Bowel Dis. 2012;18(2):372-90.

15. Valdes AM, et al. Role of the gut microbiota in nutrition and health. Br Med J. 2018;361.

16. Rajilic-Stojanovic M, et al. Phylogenetic analysis of dysbiosis in ulcerative colitis during remission. Inflamm Bowel Dis. 2013;19(3):481-8.

17. Bajaj JS, Betrapally NS, Hylemon PB, Heuman DM, Daita K, White MB, et al. Salivary microbiota reflects changes in gut microbiota in cirrhosis with hepatic encephalopathy. Hepatology. 2015;62(4):1260-71.

18. Francino MP. Antibiotics and the human gut microbiome: dysbioses and accumulation of resistances. Front Microbiol. 2016;6.

19. Bajaj, J.S., et al., Fungal dysbiosis in cirrhosis. Gut, 2018. 67(6): p. 1146-1154. This is the first study which characterises the presence of fungal dysbiosis as being important in determiming outcomes in cirrhosis.

20. David LA, Maurice CF, Carmody RN, Gootenberg DB, Button JE, Wolfe BE, et al. Diet rapidly and reproducibly alters the human gut microbiome. Nature. 2014;505:559-63.

21. Cotillard A, et al. Dietary intervention impact on gut microbial gene richness. Nature. 2013;500:585-8.

22. Singh RK, Chang HW, Yan D, Lee KM, Ucmak D, Wong K, et al. Influence of diet on the gut microbiome and implications for human health. J Transl Med. 2017;15(1):73.

23. Islam $\mathrm{KB}$, et al. Bile acid is a host factor that regulates the composition of the cecal microbiota in rats. Gastroenterology. 2011;141(5):1773-81.

24. Yokota A, Fukiya S, Islam KBMS, Ooka T, Ogura Y, Hayashi T, et al. Is bile acid a determinant of the gut microbiota on a high-fat diet? Gut Microbiobes. 2012;3(5):455-9.

25. Kastorini CM, Milionis HJ, Esposito K, Giugliano D, Goudevenos JA, Panagiotakos DB. The effect of Mediterranean diet on metabolic syndrome and its components: a meta-analysis of 50 studies and 534,906 individuals. J Am Coll Cardiol. 2011;57(11):1299 313.

26. Anania C, Perla FM, Olivero F, Pacifico L, Chiesa C. Mediterranean diet and nonalcoholic fatty liver disease. World $\mathrm{J}$ Gastroenterol. 2018;24(19):2083-94.

27.• Bajaj JS et al., Diet affects gut microbiota and modulates hospitalisation risk differentially in an international cirrhosis cohort. Hepatology, 2018. 68(1): p. 234-247. This is an important study which shows how important diet is in patients with cirrhosis in determining outcomes. The Mediterranean diet is particularly beneficial.

28. Fredua-Agyeman, M. and S. Gaisford, Assessing inhibitory activity of probiotic culture supernatants against Pseudomonas aeruginosa: a comparative methodology between agar diffusion, broth culture and microcalorimetry. World Journal of Microbiology \& Biotechnology, 2019. 35(3)

29. Dodoo CC, Stapleton P, Basit AW, Gaisford S. Use of a waterbased probiotic to treat common gut pathogens. Int J Pharm. 2019;556:136-41.

30. Ritchie, M.L. and T.N. Romanuk, A meta-analysis of probiotic efficacy for gastrointestinal diseases. PLoS One, 2012. 7(4).

31. Lee, H.L., et al., Targeted approaches for in situ gut microbiome manipulation. Genes, 2018. 9(7).

32. McLoughlin RF, et al. Short-chain fatty acids, prebiotics, synbiotics, and systemic inflammation: a systematic review and meta-analysis. Am J Clin Nutr. 2017;106(3):930-45.
33. Saab S, Suraweera D, Au J, Saab EG, Alper TS, Tong MJ. Probiotics are helpful in hepatic encephalopathy: a meta-analysis of randomized trials. Liver Int. 2016;36(7):986-93.

34. Ma YY, Li L, Yu CH, Shen Z, Chen LH, Li YM. Effects of probiotics on nonalcoholic fatty liver disease: a meta-analysis. World J Gastroenterol. 2013;19(40):6911-8.

35. Bajaj, J.S., et al., Modulation of the metabiome by rifaximin in patients with cirrhosis and minimal hepatic encephalopathy. PLoS One, 2013. 8(4).

36. Hudson, M., et al., The impact of rifaximin-alpha on the hospital resource use associated with the management of patients with hepatic encephalopathy: a retrospective observational study (IMPRESS). Frontline Gastroenterology, 2017. 8(4): p. 243-251. This study showed how manipulation of the gut microbiome with a non-absorbable antibiotic could represent significant cost saving to the healthcare system.

37.• Salehi, S., et al., Rifaximin reduces the incidence of spontaneous bacterial peritonitis, variceal bleeding and all-cause admissions in patients on the liver transplant waiting list. Alimentary Pharmacology \& Therapeutics, 2019. 50(4): p. 435-441. This retrospective study showed for the first time that manipulation of the gut microbiome with rifaximin could reduce all cirrhotic decompensating events and reduce all-cause admissions.

38. Esfeh JM, et al. Impact of pretransplant rifaximin therapy on postliver transplant infections. Am J Transplant. 2012;12:209-10.

39.• Chang, J.Y., et al., Emergence of rifampin-resistant staphylococci after rifaximin administration in cirrhotic patients. Plos One, 2017. 12(10). This study highlighted the very real concern that even short-term rifaximin use could induce antimicrobial resistance in cirrhotic populations.

40. Eiseman B, et al. Fecal enema as an adjunct in the treatment of pseudomembranous enterocolitis. Surgery. 1958;44(5):854-9.

41. El-Salhy, M., et al., Efficacy of faecal microbiota transplantation for patients with irritable bowel syndrome in a randomised, doubleblind, placebo-controlled study. Gut, 2019: p. gutjnl-2019-319630.

42. Anderson JL, Edney RJ, Whelan K. Systematic review: faecal microbiota transplantation in the management of inflammatory bowel disease. Aliment Pharmacol Ther. 2012;36(6):503-16.

43. Vrieze A, Van Nood E, Holleman F. Transfer of intestinal microbiota from lean donors increases insulin sensitivity in individuals with metabolic syndrome (vol 143, pg 913, 2012). Gastroenterology. 2013;144(1):250.

44. Mullish BH, Quraishi MN, Segal JP, McCune VL, Baxter M, Marsden GL, et al. The use of faecal microbiota transplant as treatment for recurrent or refractory Clostridium difficile infection and other potential indications: joint British Society of Gastroenterology (BSG) and Healthcare Infection Society (HIS) guidelines. Gut. 2018;67(11):1920-41.

45. Wilson, B.C., Vatanen T., Cutfield W.S., O'Sullivan J.M., The super-donor phenomenon in fecal microbiota transplantation Front Cell Infect Microbiol, 2019. 9: p. 2. This study shows that not all FMT donors are equal and some donors may be better than others.

46.• Bajaj JS, et al. Fecal microbiota transplant from a rational stool donor improves hepatic encephalopathy: a randomized clinical trial. Hepatology, 2017. 66(6): p. 1727-1738. This was the first published FMT pilot study in patients with cirrhosis showing it to be safe and potentially efficacious. .

47.• Bajaj, J.S., et al., Antibiotic-associated disruption of microbiota composition and function in cirrhosis is restored by fecal transplant. Hepatology, 2018. 68(4): p. 1549-1558. This adjunct manuscript to the study in ref. 46 showed that FMT has the potential to restore antibiotic-induced gut dysbiosis in cirrhosis.

48.• Bajaj, J.S., et al., Long-term outcomes of fecal microbiota transplantation in patients with cirrhosis. Gastroenterology, 2019. 
156(6): p. 1921-1923.e3. This follow-up study showed that FMT remains safe at 15 months post FMT in cirrhosis.

49.• Liu, R.P., et al., Neuroinflammation in murine cirrhosis is dependent on the gut microbiome and is attenuated by fecal transplant. Hepatology, 2020. 71(2): p. 611-626. This seminal study showed that FMT given to patients with cirrhosis may reduce cerebral inflammation.

50. Moss EL, Falconer SB, Tkachenko E, Wang M, Systrom H, Mahabamunuge $\mathrm{J}$, et al. Long-term taxonomic and functional divergence from donor bacterial strains following fecal microbiota transplantation in immunocompromised patients. PLoS One. 2017;12(8):e0182585.

51.• Bajaj, J.S., et al., Fecal microbial transplant capsules are safe in hepatic encephalopathy: a phase 1, randomized, placebocontrolled trial. Hepatology, 2019. 70(5): p. 1690-1703. This phase 1 study showed for the first time that encapsulated FMT is safe in cirrhosis and has efficacy. These findings now need to be confirmed in large-scale randomised clinical trials.

52.• Duan, Y., et al., Bacteriophage targeting of gut bacterium attenuates alcoholic liver disease. Nature, 2019. 575(7783): p. 505-511. This ground-breaking study suggested that manipulating the gut microbiome using a phage therapy may be able to reduce liver injury.

53. Kootte, R.S., et al., Improvement of insulin sensitivity after lean donor feces in metabolic syndrome is driven by baseline intestinal microbiota composition. Cell Metabolism, 2017. 26(4): p. 611-+.

54. Mizuno S, Masaoka T, Naganuma M, Kishimoto T, Kitazawa M, Kurokawa S, et al. Bifidobacterium-rich fecal donor may be a positive predictor for successful fecal microbiota transplantation in patients with irritable bowel syndrome. Digestion. 2017;96(1):2938.
55. Broecker, F., et al., Long-term changes of bacterial and viral compositions in the intestine of a recovered Clostridium difficile patient after fecal microbiota transplantation. Cold Spring Harbor Molecular Case Studies, 2016. 2(1).

56. Moayyedi, P., et al., Fecal microbiota transplantation induces remission in patients with active ulcerative colitis in a randomized controlled trial. Gastroenterology, 2015. 149(1): p. 102-+.

57. Anand R, Song Y, Garg S, Girotra M, Sinha A, Sivaraman A, et al. Effect of aging on the composition of fecal microbiota in donors for FMT and its impact on clinical outcomes. Dig Dis Sci. 2017;62(4): 1002-8.

58. DeFilipp M.D et al., Drug-resistant E. coli bacteremia transmitted by fecal microbiota transplant. N Engl J Med, 2019. 381: p. 2043 2050.

59. Albillos A, de Gottardi A, Rescigno M. The gut-liver axis in liver disease: pathophysiological basis for therapy. J Hepatol. 2020:72(3):558-77.

60. Santiago-Rodriguez, T.M. and E.B. Hollister, Human virome and disease: high-throughput sequencing for virus discovery, identification of phage-bacteria dysbiosis and development of therapeutic approaches with emphasis on the human gut. Viruses, 2019. 11(7).

61. Woodhouse, C.A., et al., PROFIT, a PROspective, randomised placebo controlled feasibility trial of Faecal mIcrobiota Transplantation in cirrhosis: study protocol for a single-blinded trial. BMJ Open, 2019. 9(2).

Publisher's Note Springer Nature remains neutral with regard to jurisdictional claims in published maps and institutional affiliations. 